\title{
Pancreas-protective effect of rituximab for acute-onset type 1 diabetes in the honeymoon period: a case report
}

\author{
Akira Kurozumi', Yosuke Okada1, Tadashi Arao1,2, Yusuke Miyazaki', \\ Maiko Yoshikawa', Keiichi Torimoto', Satoshi Kubo', Shingo Nakayamada' and \\ Yoshiya Tanaka' \\ ${ }^{1}$ First Department of Internal Medicine, School of Medicine, University of Occupational and \\ Environmental Health, Japan, Kitakyushu, Japan, 2Department of Internal Medicine, Japan Labour \\ Health and Welfare Organization, Kyushu Rosai Hospital, Moji Medical Center, Kyushu, Japan
}

Correspondence should be addressed to Y Tanaka

Email

tanaka@med.uoeh-u.ac.jp

\section{Summary}

A randomized controlled study of rituximab demonstrated that the drug protects pancreatic function in patients with acute-onset type 1 diabetes mellitus (AOT1DM). However, the mechanism of this protective effect is poorly understood. We examined the effects of rituximab in two patients with AOT1DM in the honeymoon period and the mechanism of these effects. Case 1 was a 40-year-old man and Case 2 was a 45 -year-old man, both diagnosed with AOT1DM. Various tests indicated intact capacity for endogenous insulin secretion and that they were in the honeymoon phase of AOT1DM. Treatment with rituximab protected against pancreatic $\beta$-cell damage and maintained somewhat the endogenous insulin secretion. In Case 2, HbA1c level was maintained below $6.5 \%$ up to 24 months after treatment. However, in Case 1, the patient showed a gradual increase in $\mathrm{HbA1c}$ level starting around 9 months but fell at 12 months to $>9.0 \%$ and required an insulin dose about twice greater than that of Case 2 . High spleen tyrosine kinase (SYK) levels were recorded in the two patients before rituximab administration and after the treatment, the levels were further increased in Case 1, but decreased in Case 2. Both patients require continuous careful follow-up for glycemic control, insulin secretion capacity, and adverse reactions in the future. Although the clinical relevance of high SYK levels in AOT1DM patients remains unclear, the difference in the change in SYK level between the two patients may explain the different clinical courses.

\section{Learning points:}

- We described the pancreas-protective effect of rituximab in two patients with acute-onset type 1 diabetes mellitus in the honeymoon period and investigated the possible mechanism of action.

- The present study demonstrated that treatment with rituximab maintained endogenous insulin secretion capacity for 2 years in the two patients.

- The phosphorylated-spleen tyrosine kinase ( $p-S Y K)$ data suggest that the differences in $\mathrm{HbA1}$ c level and the required insulin dose between the two patients could be due to reactivation or nonreactivation of $\beta$-cells. 


\section{Background}

Acute-onset type 1 diabetes mellitus (AOT1DM) is associated with the reduction in endogenous insulin secretion soon after the onset, often making glycemic control difficult. Rituximab, an anti-CD20 monoclonal antibody that depletes $\beta$-cells (1), is reported to be useful for the treatment of autoimmune diseases, hematologic diseases (e.g. idiopathic thrombocytopenic purpura and non-Hodgkin's lymphoma), and various other diseases $(2,3)$. With regard to type 1 diabetes mellitus (T1DM), a randomized controlled study of rituximab in the United States demonstrated that the drug has a protective effect on pancreatic function soon after the onset of AOT1DM (4); however, no similar studies have been reported in Japan and the mechanism of the protective effect of rituximab is poorly understood. In this studuy, we examined the pancreas-protective effect of rituximab in two patients with AOT1DM in the honeymoon period 1 month after the onset and investigated the possible mechanism of action.

\section{Case presentation}

\section{Case 1}

The patient was a 40-year-old man who presented with excessive thirst, polydipsia, polyuria, and weight loss of $10 \mathrm{~kg}$ over a 2-month period. He was diagnosed with DM and referred to our hospital for further examination. Although arterial blood gas analysis indicated no evidence of ketoacidosis, blood and urine analyses showed the presence of ketone bodies and DM with poor glycemic control (hemoglobin A1c (HbA1c) level, 9.8\%; postprandial plasma glucose level, $227 \mathrm{mg} / \mathrm{dL}$ ). Although the patient was negative for glutamic acid decarboxylase (GAD) antibody, an islet antigen (IA)-2 antibody test was performed due to ketosis and negative family history of DM. The patient was positive for IA-2 antibody, with an initial titer of $1.7 \mathrm{U} / \mathrm{mL}$, and was, therefore, diagnosed with T1DM. Accordingly, intensive insulin therapy was initiated, and a favorable glycemic control was achieved with a combination of bolus insulin (glulisine) at breakfast, lunch, and dinner (5-3-2 $\mathrm{U}$ ) and basal insulin (glargine) in the morning (1-0-0 U). The patient was discharged from the hospital after the control of T1DM.

\section{Case 2}

This was a 45-year-old man who presented with excessive thirst, polydipsia, polyuria, and weight loss of
$8 \mathrm{~kg}$ over the preceding month. He was diagnosed with DM and referred to our hospital for further examination. Although arterial blood gas analysis indicated no evidence of ketoacidosis, blood and urine analyses showed the presence of ketone bodies and diabetes with poor glycemic control (HbA1c level, 11.3\%; fasting plasma glucose level, $363 \mathrm{mg} / \mathrm{dL}$ ). He was diagnosed with T1DM based on the absence of past history of obesity, negative family history of $\mathrm{DM}$, and positivity for GAD antibody (initial titer: $29.3 \mathrm{U} / \mathrm{mL}$ ). Treatment consisted of intensive protocol of a combination of bolus insulin (lispro) at breakfast, lunch, and dinner (5-3-2 U) and basal insulin (glargine) in the morning (7-0-0 U), which resulted in favorable glycemic control. The patient was discharged from the hospital after achieving control of T1DM.

\section{Investigation}

Urinary C-peptide immunoreactivity (CPR) was $34.2 \mu \mathrm{g} /$ day (Case 1) and $28.7 \mu \mathrm{g} /$ day (Case 2), indicating intact capacity for endogenous insulin secretion and that the patients were in the honeymoon phase.

\section{Flow cytometric analysis}

Peripheral blood mononuclear cells (PBMCs) from two AOT1DM patients were isolated from peripheral blood using lymphocyte separation medium (ICN/ Cappel Pharmaceuticals, Aurora, OH, USA). For surface and intracellular staining, $2 \times 10^{5}$ cells of PBMCs, which were acquired after strict deletion of dust by threshold adjustment, were subjected to FACS analysis. PBMC were fixed with PBS containing $1 \%$ formaldehyde and permeabilized with PBS containing $0.1 \%$ saponin (saponin-PBS). After washing, they were resuspended in saponin-PBS and stained with mouse anti-human phospho-SYK (pY348) mAb (BD Pharmingen, Franklin Lakes, NJ, USA), followed by washing with saponin-PBS. PE-labeled goat anti-mouse IgG pAb (BD Pharmingen, Franklin Lakes, NJ, USA) was used as a secondary Ab. After washing by saponin-PBS, they were stained with fluorescein isothiocyanate (FITC)-labeled mouse antihuman CD19 (BD Pharmingen, Franklin Lakes, NJ, USA) antibodies. The expression levels of p-SYK in CD19+ $\beta$-cells were analyzed on a flow cytometer (FACSVerse; BD). The cells were collected and analyzed using FlowJo software (Tree Star). We defined p-SYK-positive CD19+ $\beta$-cells as cells stained higher than background staining with $\operatorname{IgG}$ control antibody. 


\section{Treatment}

They were readmitted 1 month after the initial hospital discharge for suppression of $\beta$-cell antibody production by the rituximab-mediated depletion of $\beta$-cells. The aim of rituximab therapy was to induce immunosuppression and protect against pancreatic $\beta$-cell damage. The use of rituximab was approved by the Ethics Review Committee of our university (\#11-002) and registered with the University Hospital Medical Information Network (UMIN) (\#UMIN000013622). Each patient signed an informed consent form before the treatment. Treatment with rituximab at a dose of $500 \mathrm{mg}$ (approximately $300 \mathrm{mg} / \mathrm{m}^{2}$ ) on two occasions at 1-week interval did not cause any side effects, including infusion reactions or infection.

\section{Outcome and follow-up}

In Case 1, blood glucose levels remained stable at 6 months after hospital discharge (Fig. 1). At 6 months, $\mathrm{HbA1c}$ level is $6.9 \%$, and the daily insulin requirement is $6 \mathrm{U}$, indicating stable glycemic control. In Case 2, the patient had no hypoglycemia 12 months after the onset and HbA1c level was less than 6.5\%. However, HbA1c level started to increase gradually in Case 1 around 9 months after the onset despite the good lifestyle habits, then deteriorated to $>9.0 \%$ at 12 months.

Endogenous insulin secretion capacity was evaluated using a meal test (total $450 \mathrm{kcal}$, carbohydrate $51.4 \%$, fat $33.3 \%$, and protein $15.3 \%$. We measured CPR at fasting, and after 1,2,3, and $4 \mathrm{~h}$ ). The area under the curve (AUC) of CPR was relatively maintained in both patients at 1 ,

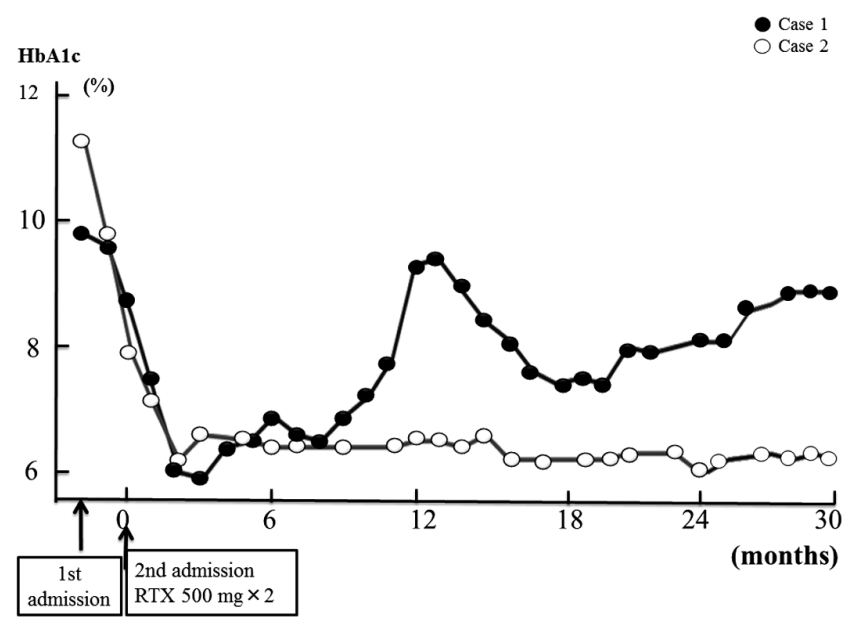

Figure 1

Serial changes in $\mathrm{HbA} 1 \mathrm{c}$ level during hospitalization and at follow-up. After readmission, rituximab was administered on two occasions 1 week apart.
Table 1 Serial changes in area under the curve (AUC) of C-peptide immunoreactivity (CPR) during hospitalization and follow-up.

\begin{tabular}{|c|c|c|c|c|c|}
\hline & \multicolumn{5}{|c|}{ AUC of CPR at follow-up (month) } \\
\hline & 0 & 1 & 3 & 6 & 12 \\
\hline Case 1 & 21.69 & 13.88 & 15.44 & 12.96 & 18.27 \\
\hline Case 2 & 11.14 & 11.34 & 10.1 & 11.39 & 6.99 \\
\hline
\end{tabular}

3 , 6, and 12 months (Table 1). The CPR index was also maintained up to 24 months (Table 2).

Figure 2 shows changes in the insulin dose. The required insulin dose was small in both patients until 9 months after rituximab treatment. However, the dose had to be increased gradually in Case 1 starting around 9 months. At 30 months after the treatment, the insulin dose in Case 1 was about double than that required for Case 2.

As shown in Fig. 3, the level of phosphorylated-spleen tyrosine kinase (p-SYK) was high in these two patients before treatment with rituximab. The p-SYK level increased again in Case 1, whereas it was inhibited in Case 2. At 24 months after the start of rituximab therapy, the p-SYK level was still high in Case 1.

\section{Discussion}

The present study describes only two patients and lacked control subjects; nevertheless, the results showed that rituximab treatment maintained the AUC of CPR and CPR index to some extent. In this regard, Pescovitz et al. (4) reported that 50 patients with AOT1DM who were treated with rituximab showed a significantly greater pancreasprotective effect 12 months after the treatment, compared with the placebo group. However, they did not observe significant difference between the rituximab and placebo groups at 24 months (5). Interestingly, 24 months after treatment, the HbA1c level and required insulin dose were significantly lower in the rituximab group. In the placebo group, the AUC of CPR was lower by approximately $40 \%$ at 12 months and $60 \%$ at 24 months. In a study by Herold and coworkers (6) that used the meal tolerance test, AUC of CPR was lower by approximately $50 \%$ at 12 months in AOT1DM patients.

Table 2 Serial changes in C-peptide immunoreactivity (CPR) index during hospitalization and at follow-up.

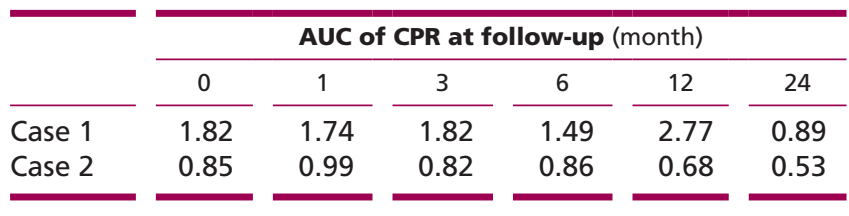




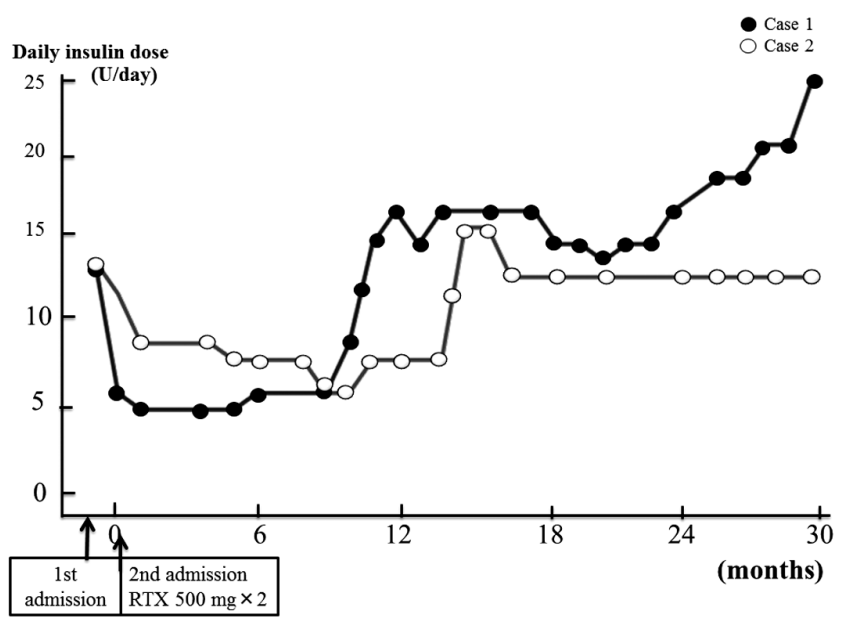

Figure 2

Serial changes in daily insulin requirement during hospitalization and at follow-up.

Rituximab exerts its effects through the suppression of $\beta$-cell antibody production and depletion of $\beta$-cells. $\mathrm{Xu}$ and coworkers (7) reported that AOT1DM patients treated with rituximab showed a significant decrease in IA-2 antibody concentration but no changes in GAD antibody titer 4 months after the treatment. In the present study, no significant changes in GAD antibody were observed in Case 2, whereas IA-2 antibody expression became negative 6 months later in Case 1 . However, both the study of $\mathrm{Xu}$ and the present study did not include a control group, and there was no correlation between changes in auto-antibodies and endogenous insulin secretion capacity or glycemic control. Therefore, whether the changes in the expression levels of auto-antibodies correlate with the control of disease progression remains unclear. However, it has been suggested that in multiple sclerosis (8) and T1DM $(4,5,6)$, rituximab may suppress $\mathrm{T}$ cells through the suppression of the antigen-presenting function of $\beta$-cells. Follicular helper $\mathrm{T}$ (Tfh) cells play an important role in the maturation and antibody production of $\beta$-cells (9). The number of Tfh cells is reportedly high in T1DM patients, their count correlates significantly and negatively with fasting CPR level, and treatment with rituximab reduces their number and IL6 and IL21 levels in peripheral blood (7). Although there was no correlation between changes in Tfh cell count and those in CPR level, these findings may help to elucidate the mechanism of the protective effect of rituximab on pancreatic function. In the two patients under investigation, $\mathrm{T}$ cells were evaluated by lymphocyte analysis, and no apparent changes were observed after rituximab treatment. However, a longer follow-up period is necessary for a more accurate assessment.

SYK, a nonreceptor tyrosine kinase, is activated by autophosphorylation, resulting in signal transduction to the downstream. SYK plays an important role in the differentiation and activation of mast cells, macrophages, and osteoclasts, in addition to $\beta$-cells (10). Several SYK inhibitors have been introduced in recent years, and their efficacy in the treatment of allergic rhinitis and rheumatoid arthritis (RA) has been attracting attention (11). PRT062607, a novel SYK inhibitor, has been shown to inhibit the kinase activity of SYK as an ATP-competitive inhibitor. It inhibits $\beta$-cell receptor stimulation in a dosedependent manner. Oral administration of this drug is effective in animal models of RA (12). As shown in Fig. 3, the p-SYK level increased again in Case 1, and this was associated with poor clinical course 6 months later, whereas it was inhibited in Case 2, which was associated

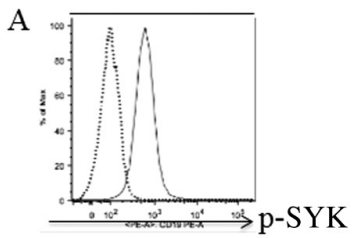

$\Delta \mathrm{MFI}=1059$

B

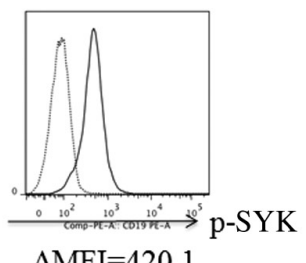

$\triangle \mathrm{MFI}=420.1$

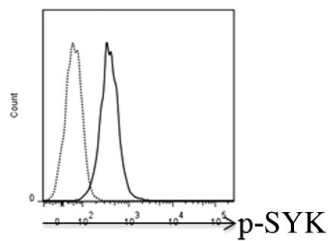

$\Delta \mathrm{MFI}=361$

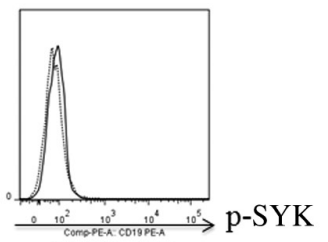

$\Delta \mathrm{MFI}=5.7$

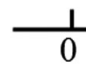

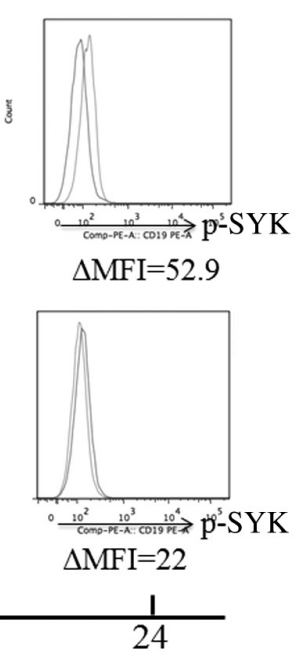

(Months)
Figure 3

Serial changes in phosphorylation of SYK in CD19+ $\beta$-cells of Case 1 (A) and Case 2 (B) during hospitalization and at follow-up. Open histograms depict the number of cells stained with anti-human phospho-SYK (pY348) mAb in a logarithmic scale on a fluorescence amplifier. Dotted histograms show results obtained with IgG control mAb as a negative control. 
with favorable clinical course. In an experimental study, early treatment of nonobese diabetic (NOD) mice with SYK inhibitors significantly inhibited the onset of T1DM (13). Although the clinical significance of high p-SYK levels in AOT1DM patients remains clear, the difference in the changes in p-SYK level between the two patients may explain the different subsequent clinical courses.

Pescovitz et al. $(4,5)$ reported infusion reactions in many patients after the initial rituximab infusion, but these symptoms improved after the second infusion. The two patients in our report showed no infusion reactions, such as fever, hypotension, tachycardia, or pruritus, with acetaminophen, glucocorticoids, and antihistamine drug after the first or the second dose of rituximab. Although serum IgM level decreased from 108 to $67 \mathrm{mg} / \mathrm{dL}$ in Case 1, and from 68 to $36 \mathrm{mg} / \mathrm{dL}$ in Case 2, as measured at 24 months after the completion of treatment, similar to the findings in previous studies $(4,5)$, there were no marked changes in serum IgG and IgA levels, and no infections were detected.

Despite the lack of a control group, the present study demonstrated that treatment with rituximab maintained endogenous insulin secretion capacity for 2 years in the two patients. In addition, the p-SYK data suggest that the differences in HbA1c level and the required insulin dose between the two patients could be due to reactivation or nonreactivation of $\beta$-cells. Both patients require continuous and close follow-up for glycemic control, insulin secretion capacity, and appearance of adverse reactions in the future. Many issues remain, such as the establishment of safety and the cost of rituximab treatment. Randomized clinical control trials await to be performed in Japan to determine the efficacy of rituximab in the treatment of AOT1DM.

\section{Declaration of interest}

The authors declare that there is no conflict of interest that could be perceived as prejudicing the impartiality of research reported.

\section{Funding}

This research did not receive any specific grant from any funding agency in the public, commercial, or not-for-profit sector.

\section{Patient consent}

Written informed consent was obtained from the patients for publication of this case report.
Author contribution statement

All coauthors listed contributed substantially to the presentation of this manuscript.

\section{References}

1 Reff ME, Carner K, Chambers KS, Chinn PC, Leonard JE, Raab R, Newman RA, Hanna N \& Anderson DR 1994 Depletion of B cells in vivo by a chimeric mouse human monoclonal antibody to CD20. Blood 83 435-445.

2 Nielsen CH, El Fassi D, Hasselbalch HC, Bendtzen K \& Heqedus L 2007 B-cell depletion with rituximab in the treatment of autoimmune diseases. Graves' ophthalmopathy the latest addition to an expanding family. Expert Opinion on Biological Therapy 7 1061-1078. (doi:10.1517/14712598.7.7.1061)

3 Edwards JC \& Cambridge G 2006 B-cell targeting in rheumatoid arthritis and other autoimmune diseases. Nature Reviews Immunology 5 394-403. (doi:10.1038/nri1838)

4 Pescovitz MD, Greenbaum CJ, Krause-Steinrauf H, Becker DJ, Gitelman SE, Goland R, Gottlieb PA, Marks JB, McGee PF, Moran A, et al. 2009 Rituximab, B-lymphocyte depletion, and preservation of beta-cell function. New England Journal of Medicine $3612143-2152$. (doi:10.1056/NEJMoa0904452)

5 Pescovitz MD, Greenbaum CJ, Bundy B, Becker DJ, Gitelman SE, Goland R, Gottlieb PA, Marks JB, Moran A, Raskin P, et al. 2009 B-lymphocyte depletion with rituximab and $\beta$-cell function: two-year results. Diabetes Care 37 453-459. (doi:10.2337/dc13-0626)

6 Herold KC, Hagopian W, Auger JA, Poumian-Ruiz E, Taylor L, Donaldson D, Gitelman SE, Harlan DM, Xu D, Zivin RA, et al. 2002 Anti-CD3 monoclonal antibody in new-onset type 1 diabetes mellitus. New England Journal of Medicine 346 1692-1698. (doi:10.1056/NEJMoa012864)

7 Xu X, Shi Y, Cai Y, Zhang Q, Yang F, Chen H, Gu Y, Zhang M, Yu L \& Yang T 2013 Inhibition of increased circulating Tfh cell by anti-CD20 monoclonal antibody in patients with type 1 diabetes. PLOS ONE $\mathbf{8}$ e79858. (doi:10.1371/journal.pone.0079858)

8 Hauser SL, Waubant E, Arnold DL, Vollmer T, Antel J, Fox RJ, Bar-Or A, Panzara M, Sarkar N, Agarwal S, et al. 2008 B-cell depletion with rituximab in relapsing-remitting multiple sclerosis. New England Journal of Medicine 358 676-688. (doi:10.1056/ NEJMoa0706383)

9 Moon JJ, Suh H, Li AV, Ockenhouse CF, Yadava A \& Irvine DJ 2012 Enhancing humoral responses to a malaria antigen with nanoparticle vaccines that expand Tfh cells and promote germinal center induction. PNAS 109 1080-1085. (doi:10.1073/ pnas.1112648109)

10 Tohyama Y \& Yamamura H 2009 Protein tyrosine kinase, syk: a key player in phagocytic cells. Journal of Biochemistry 145 267-273. (doi:10.1093/jb/mvp001)

11 Weinblatt ME, Kavanaugh A, Genovese MC, Musser TK, Grossbard EB \& Maqilavy DB 2010 An oral spleen tyrosine kinase (Syk) inhibitor for rheumatoid arthritis. New England Journal of Medicine 363 1303-1312. (doi:10.1056/NEJMoa1000500)

12 Uckun FM, Qazi S, Ma H, Tuel-Ahlgren L \& Ozer Z 2010 STAT3 is a substrate of SYK tyrosine kinase in B-lineage leukemia/lymphoma cells exposed to oxidative stress. PNAS 107 2902-2907. (doi:10.1073/ pnas.0909086107)

13 Colonna L, Catalano G, Chew C, D'Agati V, Thomas JW, Wong FS, Schmitz J, Masuda ES, Reizis B, Tarakhovsky A, et al. 2010 Therapeutic targeting of Syk in autoimmune diabetes. Journal of Immunology $\mathbf{1 8 5}$ 1532-1543. (doi:10.4049/jimmunol.1000983)

Received in final form 19 April 2016

Accepted 22 April 2016 